\title{
Uncertainties in Adoption of Model-Based Definition and Enterprise for High-Value Manufacturing
}

\author{
Kamran GOHER ${ }^{\mathrm{a}, 1}$, Essam SHEHAB ${ }^{\mathrm{b}}$, Ahmad AL-ASHAAB $^{\mathrm{a}}$ and Shoaib SARFRAZ ${ }^{\mathrm{a}}$ \\ ${ }^{a}$ Manufacturing Department, School of Aerospace, Transport and Manufacturing, \\ Cranfield University, Cranfield, Bedfordshire, MK43 0AL, UK \\ ${ }^{b}$ Mechanical and Aerospace Engineering Department, School of Engineering and \\ Digital Sciences, Nazarbayev University, Nur-Sultan 010000, Kazakhstan
}

\begin{abstract}
The world is adopting digital technologies at a rapid pace which are the key enablers to improve every walk of life in the modern era. This quest for digitization has equal rigor within the manufacturing industry. $2 \mathrm{D}$ drawings have been used historically to define the specifications of a product for manufacturing. The evolution in digital technologies has made it possible to improve the way of representing these specifications in the form of $3 \mathrm{D}$ models which is known as Model-Based Definition (MBD). However, the 2D representation is still the authoritative source within the industry for engineering definition and related documents in the product lifecycle. Though MBD has been adopted in design, discrete part manufacturing, and inspection stages to some extent, the industry heavily relies on the conventional $2 \mathrm{D}$ representation of product definition. The digitization process of product definition lies within the adoption of MBD as the authoritative source for all the enterprise activities which is referred to as Modelbased Enterprise (MBE). However, there are several uncertainties and risks in this process. In this work, it is ascertained to formulate the key uncertainties in the transformation towards MBD/E. These uncertainties are categorized and analyzed further for visualization.
\end{abstract}

Keywords. Model-Based Definition, Model-Based Enterprise, Uncertainties, Digital Manufacturing, Digital Thread, Digital Transformation

\section{Introduction}

Model-Based Definition (MBD) is a 3D digital model for product definition that covers all the product information and thus replaces the conventional $2 \mathrm{D}$ drawings [1]. The concept of Model-Based Enterprise (MBE) lies in employing model-based definition as the single authoritative data source for all the engineering activities throughout the product lifecycle. This involves working with all internal and external stakeholders that use the product data including the suppliers. Additionally, the model is created once and then reused for all the activities [2-4]. The traditional 2D representation has been used for a long period with well-established standards and mature practices within the industry. The other downstream documents like assembly instructions, MRO (maintenance, repair, and overhaul) instructions are also based on these drawings. The preparation and

${ }^{1}$ Corresponding Author. k.goher@cranfield.ac.uk 
consumption of these drawings and the related documents need considerable time and knowledge of the designer and the consumer of the product definition. The introduction of computer-aided design (CAD) applications made it possible to enhance the visualization of geometry by the introduction of $3 \mathrm{D}$ models. This evolution took a further step beyond visualization by adding functional tolerance and annotations. Thus, the 3D digital model started offering the capabilities to partially replace 2D drawings. The recent technologies are allowing semantic information embedded with the model so that these become machine-consumable. The future of this digital definition of the product is aimed at carrying product information from all the lifecycle stages with semantic capabilities ensuring a digital thread. In addition to support MBE, such a product model is supposed to be at the heart of industry 4.0 and the industrial internet of things.

The aerospace and automotive sector are the early adopters of model-based definition. However, the authoritative source of product information for most internal and external processes is still the 2D drawings [5]. Though the software industry is advancing at a rapid pace by offering technological solutions, there are plenty of uncertainties that are inhibiting the realization of the model-based enterprise. Many researchers have highlighted the problems and challenges associated with this regard. However, systematic work on the uncertainties in the process of adopting model-based definition is missing. This work has addressed this gap by outlining the key uncertainties of MBD. These uncertainties are further categorized into five major categories and discussed in detail in the following sections.

\section{Understanding the uncertainty}

\subsection{Definition}

The world is in the phase of continuous transformation and becoming ever complex each passing day with the fast pace of innovation and introduction of new technologies. These advancements lead to new, unknown, and unpredictable situations that cause doubts through a lack of confidence and assurance. These situations are referred to as the uncertainties and risks which affect the decision-making process [6]. It is therefore imperative to build the capability of understanding and managing these uncertainties and risks to enhance the decision-making process for the adoption of new technologies.

\subsection{Risk and Uncertainty}

Uncertainty is the lack of complete certainty or where the exact outcome or state is not known [7]. While uncertainty can have positive, negative, or no effect, the risk is a type of uncertainty where the outcome of a process or event has always a negative effect on the project. In this work, the term uncertainty is used instead of risk, as risk is sometimes an ambiguous term. Uncertainty on the other hand focus on the sources and response options of the uncertainties in the project. And thus can be used to shape effective organizational strategy [8]. 


\section{Uncertainties of $\mathrm{MBD} / \mathrm{E}$}

\subsection{Dimensions of uncertainties in model-based definition}

In their previous work, the authors figured out the key issues and challenges in modelbased definition and enterprise [5]. After further literature study and critical brainstorming, a list of uncertainties is prepared consisting of 31 uncertainties. This work is part of a research project aiming at development of a framework for management of uncertainties in adoption of MBD in collaboration with two renowned aerospace organizations. The identification of uncertainties is the first phase out of the planned three phases of the project. These three phases are identification, assessment and response. For refinement of the list of uncertainties, semi-structured interviews are conducted with cross-domain experts within these industries. This resulted in addition of some uncertainties and deletion of some others which do not match the definition of uncertainty. This refinement process is further extended to discussions within digital manufacturing research group at Cranfield University. This resulted in fine-tuning of the terminologies and practicality of the underlying definitions and concepts.

During this process, various dimensions of were considered, which are explained as under.

- Model elements - 3D model is the base of MBD. It is important to see what should be the elements of the model that will enable a model-based enterprise to be realized as per its full conceptual form.

- Software support - Refers to the capabilities of currently available software to meet the MBE needs.

- Hardware support - Refers to the hardware capabilities in the context of consumption of semantic elements of the model-based definition.

- Cost of implementation - Cost is a critical criterion in the decision-making process. The cost of implementation was therefore considered for both internal and external stakeholders.

- Supplier readiness - Refers to the readiness of suppliers to fit in the ecosystem of the model-based enterprise.

- Data trust issues - Refers to the issues related to trustworthiness of the digital format of the data, which was previously available in its mature form in the case of $2 \mathrm{D}$ definition practices.

- Other digital data challenges - Refer to the challenges of digital data concerning model-based definition/enterprise.

\subsection{List of Uncertainties}

Through the process described above, a list of 31 uncertainty types are identified and then categorized into five categories. These uncertainties are shown in Figure 1 and are discussed in the following lines.

\subsubsection{Technological Readiness}

Uncertainty lies in the software capabilities of existing MBD solutions to meet the requirements of all the lifecycle stages in the perspective of MBE. The current capabilities have addressed design, manufacturing, and inspection needs to some extent, 
but they do not offer capabilities that could build a model that satisfies other downstream stages e.g. assembly, test, and MRO $[9,10]$.

The conventional 2D drawings are supported by mature standards, while for modelbased definition the standards are evolving and not sufficient to meet the industry needs. ASME 14.41, for example, lacks to describe which data set document or template to follow [1]. Another uncertainty lies in the lack of recommended practices for a consistent interpretation of the developed standards and their implementation $[9,11]$. There is also an uncertainty in the semantic product and manufacturing information (PMI) incorporation capabilities of the software and the consumption capabilities of the machines i.e., computer numerical control machines (CNCs), coordinate measuring machines (CMMs), and intelligent tooling. There is a lack of hardware that supports embedded PMI and other semantic data for consumption [11]. The capabilities in this regard are growing but are not fully matured to the MBE level. There are a variety of applications and data formats that are in use at this day for various domains of the product lifecycle. These are developed by software vendors which do it in their way to win a competitive advantage. In the MBE perspective, uncertainty exists in the interoperability of these data types, systems, languages, and processes [3,12]. Furthermore, the uncertainty in the availability of low-cost software and hardware technologies that can be easily adopted by the suppliers to fit in the MBE is also a matter of concern for the high-value manufacturing industry.

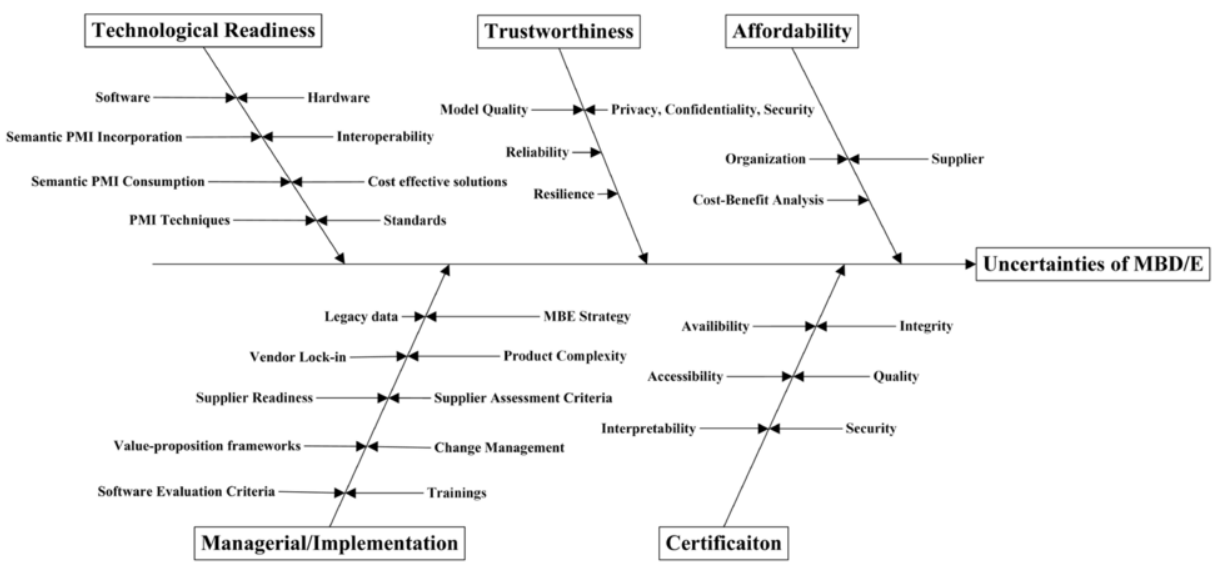

Figure 1. Uncertainties of model-based definition/enterprise

\subsubsection{Managerial / Implementation}

Applying new technology to a new product is rather easier but applying it to legacy products involves extra cost, time, and effort [12], which adds to the uncertainty of MBD in its adoption at the enterprise level. Another uncertainty lies in falling into the ecosystem of the application provider i.e., vendor lock-in. Choosing one solution will lock all the stakeholders in the ecosystem of that supplier [13]. This is quite a crucial aspect and that is where standardization efforts are eagerly needed. 
To accomplish the MBE strategy the suppliers are equally important to be proficient in technology as well as in human resource capabilities. They are also needed to be assessed for these capabilities to fit into the new ways of working. Frameworks are needed for such assessments [4]. There is also a need to establish frameworks for the value proposition of MBD for different domains of application in the product lifecycle. Furthermore, frameworks for evaluating the new MBE software applications must be established. The absence of all such frameworks would increase the probability of uncertain outcomes in the adoption of MBD.

There are concerns of industry on the ability of MBD to handle product complexity. As the product complexity rises the more difficult is to apply MBD. The organizational changes required by $\mathrm{MBE}$ are not possible at once and a phased approach is recommended for it. However, an organizational strategy that is not architected keeping all the aspects in view, is also a source of uncertainty. The MBE will be needing modified workflows and procedures [14] and corresponding training of both within and supplier end [13].

\subsubsection{Trustworthiness}

From a trustworthiness perspective, the major uncertainties lie in the privacy, confidentiality, and security of the digital format data of MBD. Another source of mistrust is a poor model quality that can be caused by the software user, poor model development technique, a system or translation error [3]. The lack of trust from the users on MBD as a replacement of conventional drawing also creates uncertainty in the MBE strategy. MBD should be reliable enough that it fulfills all the requirements of users that were previously available to them through the conventional form of definition. Another source of uncertainty is the doubt over the resilience of the available data formats to cope with the fast and frequent technological advancements $[15,16]$.

\subsubsection{Certification}

There are certain requirements of aerospace certification bodies that the design data must be secure, accessible, and available over a certain period across the lifespan of the product. This retention of the data must also ensure its integrity and quality. It must also be interpretable by all versions of the software. These conditions are termed long-term archival and retrieval $[3,10]$. There is a widespread concern across the high-value manufacturing industry over the uncertainty of model-based data to cope with these issues.

\subsubsection{Affordability}

Digital technologies offer competitive advantages but at the same time, they need sometimes huge investments. Therefore, while adopting MBD, the affordability of an organization and its suppliers for these technologies is an important factor to consider. Moreover, the lack of any framework for the cost-benefit analysis in the adoption of MBD is also a source of uncertainty in decision-making for MBE. 


\section{Conclusions and future work}

Model-based definition is seen at the core of digitization in the area of product definition and is expected to provide a competitive advantage to manufacturing organizations by improving their processes. High-value manufacturing industries such as aerospace are the early adaptors of this technology. They are also participating in further advancements by giving their regular input and feedback to academia and solution providers. However, this adoption is partial, and to physically achieve the conceptual status of MBE there is a range of uncertainties to be assessed and mitigated from the perspective of the industry. On the other hand, there are some hypes created by the promoters and the software solution providers as well. However, the purpose of this study is to assess MBD/E with a neutral perspective and figure out what are the uncertainties in the process of adoption. The key uncertainties are figured out from literature and collaboration with the industry and categorized into five distinct categories with their definitions. The future work of this project will assess these uncertainties for their relative importance and explore the mitigation strategies for highly important uncertainties. This work could enable the industry to formulate its MBE strategy effectively. At the same time, it would help academia and software solution providers find out the grey areas for future research and development in the area of MBD/E.

\section{Acknowledgment}

The authors are thankful to Higher Education Commission, Pakistan, and Cranfield University, United Kingdom for funding this research.

\section{References}

[1] V. Quintana et al., Will model-based definition replace engineering drawings throughout the product lifecycle? A global perspective from aerospace industry, Computers in Industry 61 (2010), 497-508.

[2] T. Hedberg et al., Testing the digital thread in support of model-based manufacturing and inspection, Journal of Computing and Information Science in Engineering, 16 (2016), 021001.

[3] S. Frechette, Model-based enterprise for manufacturing, In: 44th CIRP International Conference on Manufacturing Systems, Madison, WI; 2011.

[4] K. Goher et al., Model-based definition and enterprise: State-of-the-art and future trends, Proceedings of the Institution of Mechanical Engineers, Part B: Journal of Engineering Manufacture (2020), 1-12.

[5] K. Goher et al., Challenges of model-based definition for high-value manufacturing. In: 17th International Conference on Manufacturing Research, Belfast, 2019, 22-27.

[6] J. A. Erkoyuncu et al., Identifying uncertainties for industrial service delivery: a systems approach, International Journal of Production Research, 51(2013), 6295-6315

[7] D. W . Hubbard, How to measure anything finding the value of 'intangibles' in business, John Wiley \& Sons, New Jersy, 2010.

[8] S. Ward and C. Chapman, Transforming project risk management into project uncertainty management, International Journal of Project Management, 21(2003), 97-105.

[9] Minimum model-based definition (MBD) and bill of material (BOM) definition with STEP AP242 - Problem statement, direction and preliminary requirements, Aerospace and Defence PLM Action Group, 2017, https://www.cimdata.com/images/position-papers/ad-pag-mbd-bom-pp-4.0.pdf (accessed 26 April 2021) 
[10] A. M. Miller et al., Towards identifying the elements of a minimum information model for use in a model-based definition, In: Proceedings of the ASME 2017 12th International Manufacturing Science and Engineering Conference, Los Angeles, 2017.

[11] K. Fischer et al., Investigating the impact of standards-based interoperability for design to manufacturing and quality in the supply chain, National Institute of Standards and Technology, NIST GCR-15-1009.

[12] S. P. Ruemler et al., Promoting model-based definition to establish a complete product definition, Journal of Manufacturing Science and Engineering, 139(2017), 051008-1-7.

[13] J. Bijnens and D. Cheshire, The current state of model-based definition, Computer-Aided Design and Applications, 16 (2019), 308-317.

[14] C. Briggs et al., Model-Based Definition, In: 51st AIAA/ASME/ASCE/AHS/ASC Structures, Structural Dynamics, and Materials Conference, Orlando, 2010, 1-12.

[15] T. D. Hedberg et al., Method for enabling root of trust in support of product data certification and traceability, ASME Journal of Computing and Information Science in Engineering, 19(2019), 041003.

[16] T. D. Hedberg et al., Embedding X.509 digital certificates in three-dimensional models for authentication, authorization, and traceability of product data, Journal of Computing and Information Science in Engineering 17(2016), 011008-1-11 